\title{
Editorial
}

\section{Nonlinear Systems in Function Spaces and Applications in Biomedical Sciences, Control Theory, and Engineering}

\author{
Jin Liang, ${ }^{1}$ C. Cuevas, ${ }^{2}$ T. Diagana, ${ }^{3}$ G. N'Guérékata, ${ }^{4}$ and M. Van Nguyen ${ }^{5}$ \\ ${ }^{1}$ Department of Mathematics, Shanghai Jiao Tong University, Shanghai 200240, China \\ ${ }^{2}$ Departamento de Matemática, Universidade Federal de Pernambuco, 50740-540 Recife, PE, Brazil \\ ${ }^{3}$ Department of Mathematics, Howard University, 2441 6th Street NW, Washington, DC 20059, USA \\ ${ }^{4}$ Department of Mathematics, Morgan State University, 1700 E. Cold Spring Lane, Baltimore, MD 21251, USA \\ ${ }^{5}$ Department of Mathematics and Philosophy, Columbus State University, 4225 University Avenue, Columbus, GA 31907, USA
}

Correspondence should be addressed to Jin Liang; jinliang@sjtu.edu.cn

Received 27 April 2014; Accepted 27 April 2014; Published 28 May 2014

Copyright (c) 2014 Jin Liang et al. This is an open access article distributed under the Creative Commons Attribution License, which permits unrestricted use, distribution, and reproduction in any medium, provided the original work is properly cited.

\begin{abstract}
Most mathematical systems coming from real processes and phenomena, which appear in physics, population dynamics, biotechnology, medicine, and engineering, are inherently nonlinear in nature. So, various nonlinear systems constitute basic and important mathematical models of real world phenomena, and related problems are of great interest to mathematicians as well as scientists in many other fields. The study of nonlinear systems is theoretically challenging and practically interesting. It has in all the years been a major object of investigations, and the development of related research goes on quickly every day.

The purpose of this special issue is to present some new progress on the study of nonlinear systems in function spaces and applications. All the published papers in the special issue are carefully selected. From these papers, the reader will see many of the latest achievements on the following interesting topics: the finite-time stabilization problem of dynamic nonholonomic wheeled mobile robots with parameter uncertainties and discontinuous switching controller and switching control design method; the existence of solutions to a functional differential equation with statedependent delay on the basis of the space of real continuous functions on the real axis and the theories of fixed points and operator semigroups; the additivity of $D$-property of $t$-metrizable spaces and the function spaces $C_{p} X_{i}$, where each $X_{i}$ is Lindelöf $\Sigma$; the existence and the uniqueness of the positive solutions to nonlinear fractional differential
\end{abstract}

equations involving the $p$-Laplacian operators; the existence of fixed point for generalized eventual cyclic gross contractive mappings in metric spaces; damped algorithms for the split fixed point and equilibrium problems which include fixed point problems, equilibrium problems, and variational inequality problems as special cases; the existence of mild and strong solutions for semilinear evolution equations in Banach spaces with nonlocal initial conditions and applications to semilinear functional partial differential equations with nonlocal conditions; and the existence of fixed point for JHoperators and occasionally weakly biased pairs.

\section{Acknowledgments}

We thank all the authors of the 24 papers for submitting their papers to this special issue for consideration. Especially, we thank all the reviewers very much for their comments and suggestions.

Jin Liang

C. Cuevas

T. Diagana

G. N’Guérékata

M. Van Nguyen 


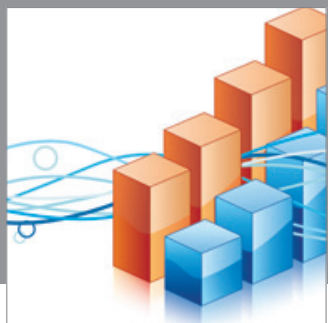

Advances in

Operations Research

mansans

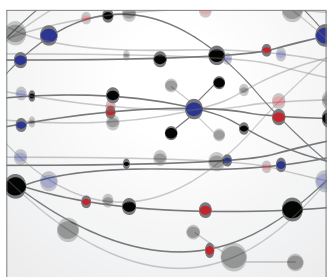

The Scientific World Journal
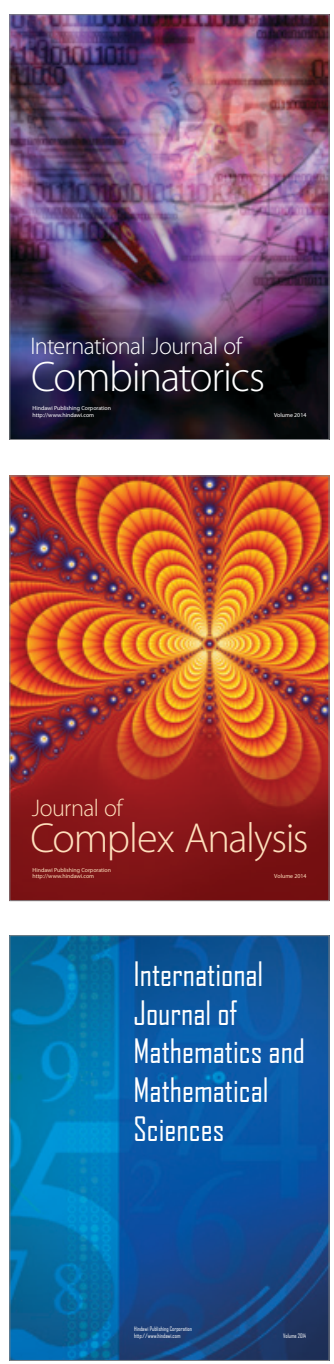
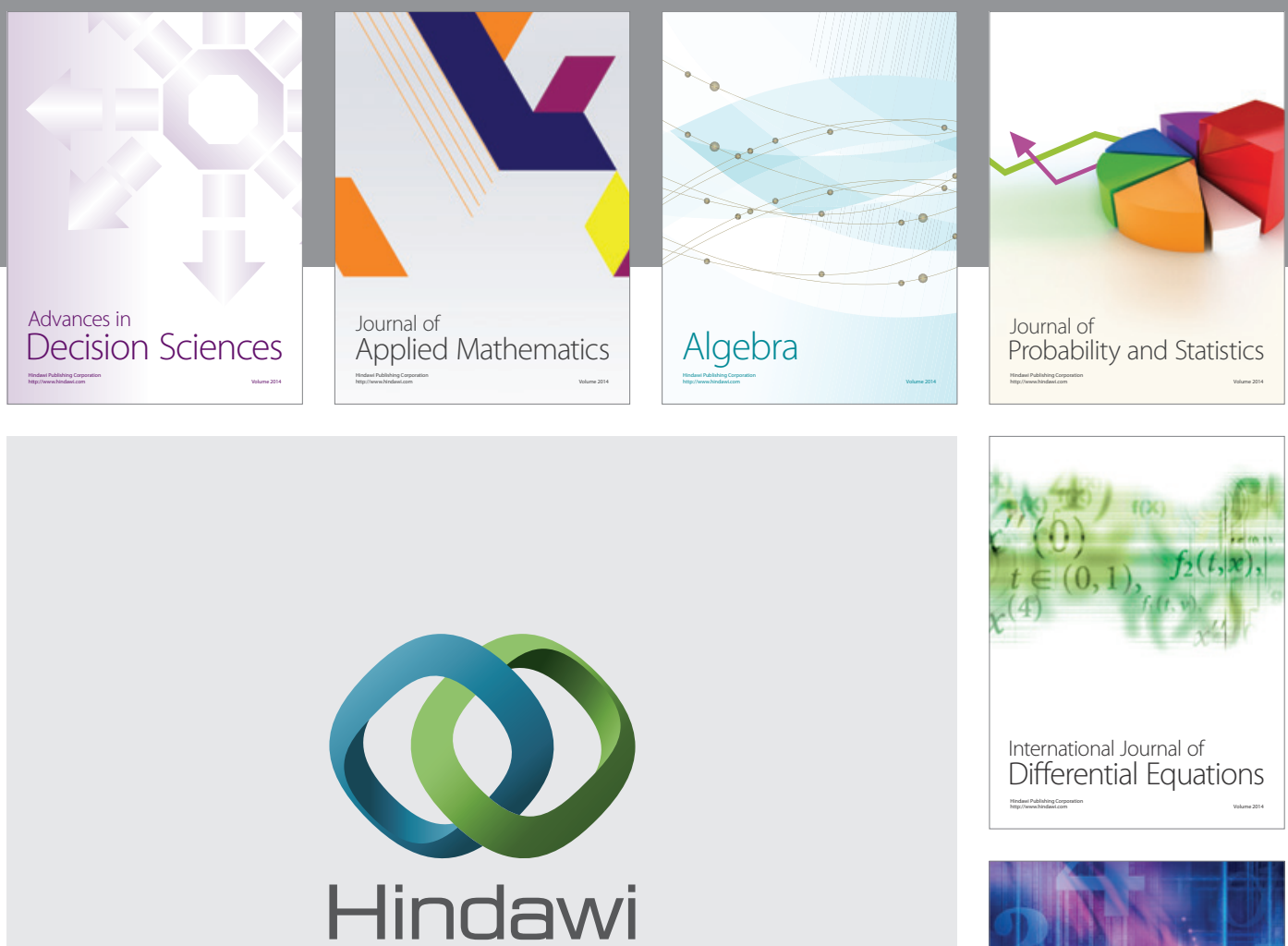

Submit your manuscripts at http://www.hindawi.com
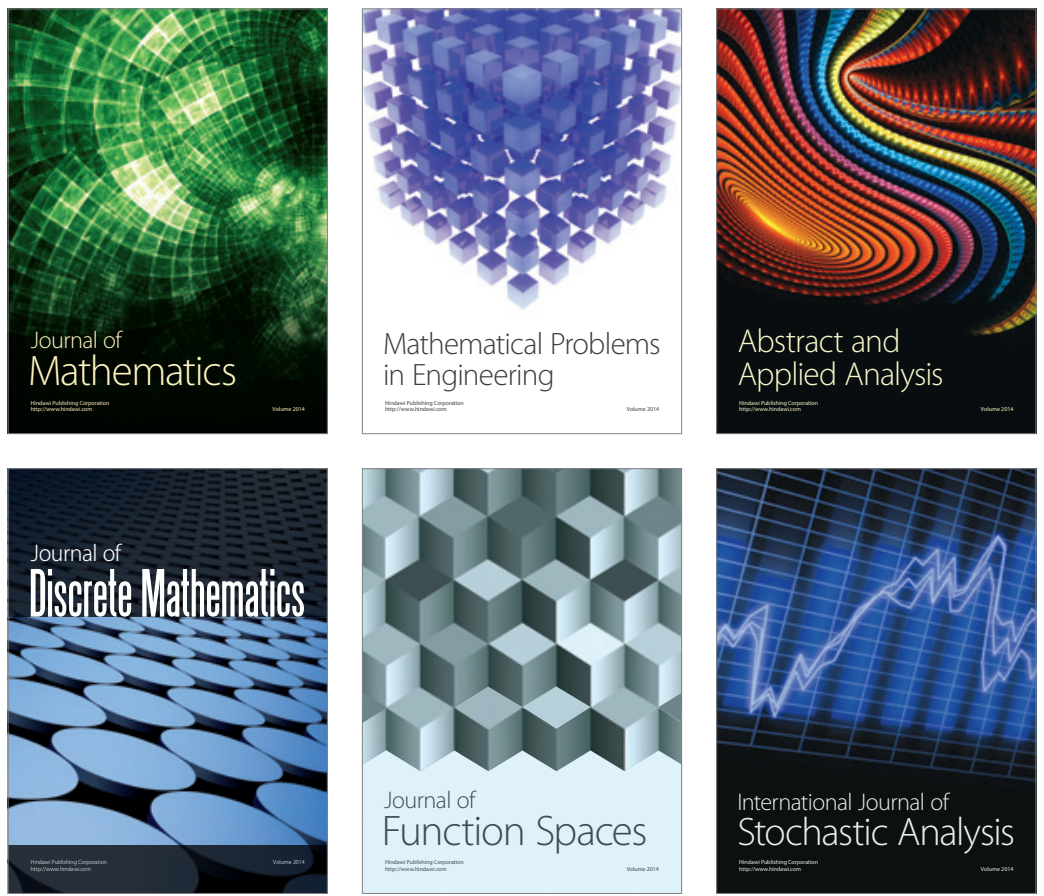

Journal of

Function Spaces

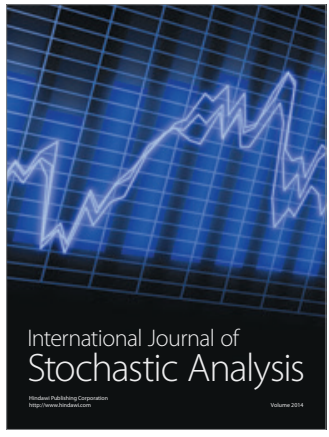

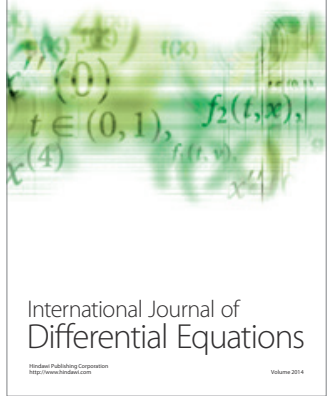
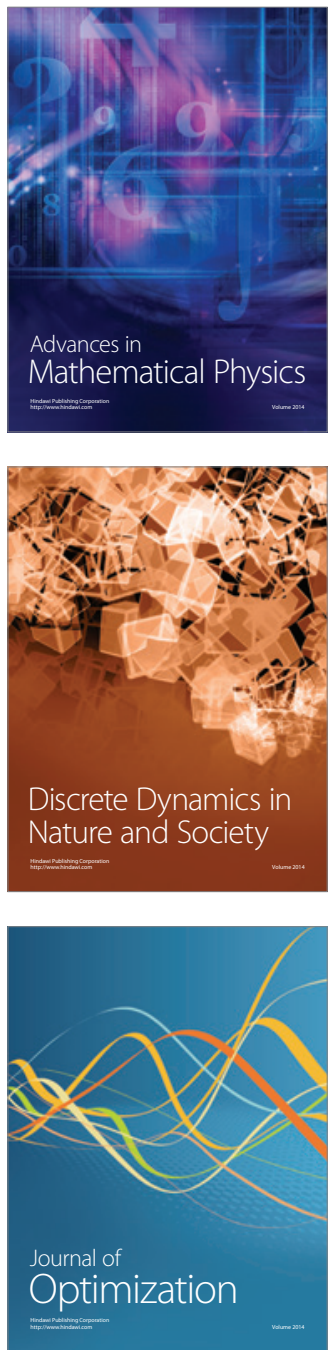